\title{
Comparison of the Legionella pneumophila population structure as determined by sequence-based typing and whole genome sequencing
}

Anthony P Underwood ${ }^{1 *}$, Garan Jones ${ }^{1,3}$, Massimo Mentasti ${ }^{2}$, Norman K Fry ${ }^{2}$ and Timothy G Harrison ${ }^{2}$

\begin{abstract}
Background: Legionella pneumophila is an opportunistic pathogen of humans where the source of infection is usually from contaminated man-made water systems. When an outbreak of Legionnaires' disease caused by L. pneumophila occurs, it is necessary to discover the source of infection. A seven allele sequence-based typing scheme (SBT) has been very successful in providing the means to attribute outbreaks of L. pneumophila to a particular source or sources. Particular sequence types described by this scheme are known to exhibit specific phenotypes. For instance some types are seen often in clinical cases but are rarely isolated from the environment and vice versa. Of those causing human disease some types are thought to be more likely to cause more severe disease. It is possible that the genetic basis for these differences are vertically inherited and associated with particular genetic lineages within the population. In order to provide a framework within which to test this hypothesis and others relating to the population biology of L. pneumophila, a set of genomes covering the known diversity of the organism is required.
\end{abstract}

Results: Firstly, this study describes a means to group L. pneumophila strains into pragmatic clusters, using a methodology that takes into consideration the genetic forces operating on the population. These clusters can be used as a standardised nomenclature, so those wishing to describe a group of strains can do so. Secondly, the clusters generated from the first part of the study were used to select strains rationally for whole genome sequencing (WGS). The data generated was used to compare phylogenies derived from SBT and WGS. In general the SBT sequence type (ST) accurately reflects the whole genome-based genotype. Where there are exceptions and recombination has resulted in the ST no longer reflecting the genetic lineage described by the whole genome sequence, the clustering technique employed detects these sequence types as being admixed, indicating their mixed inheritance.

Conclusions: We conclude that SBT is usually a good proxy for the genetic lineage described by the whole genome, and therefore utility of SBT is still suitable until the technology and economics of high throughput sequencing reach the point where routine WGS of L. pneumophila isolates for outbreak investigation is feasible.

Keywords: Legionella pneumophila, Sequence-based typing, Whole genome sequencing, Clustering, Population structure, Recombination

\footnotetext{
* Correspondence: anthony.underwood@phe.gov.uk

'Bioinformatics Unit, Microbiology Services (Colindale), Public Health

England, 61 Colindale Avenue, London NW9 5EQ, UK

Full list of author information is available at the end of the article
} 


\section{Background}

Legionellosis is acquired by inhalation or aspiration of Legionella spp. from a contaminated environmental source. Thus, when a case of legionellosis is recognized others may become infected from the same source if appropriate control measures are not taken to reduce the risk of further transmission. The source of the outbreak or incident can be determined by epidemiological investigation together with characterization of legionellae isolated from patients and putative environmental sources [1,2].

As the vast majority of cases of legionellosis are caused by Legionella pneumophila, and this species is very common in the environment, discriminatory typing methods are needed to differentiate between isolates if a convincing epidemiological link between patient and source is to be established. Consequently a large number of molecular methods have been investigated for epidemiological typing purposes and one of these, devised by members of the European Working Group for Legionella Infections (EWGLI) and termed sequence-based typing (SBT), has become established internationally as the typing method of choice [3,4]. This method is a variant of the classic multi-locus sequence typing (MLST) schemes used to identify bacterial lineages, the utility of which has been previously described [5].

The availability of a substantial quantity of international SBT typing data has led to the recognition that the majority of legionellosis is caused by a relatively small subset of all strains recovered from the environment $[6,7]$. This poses the question of whether some clonal lineages have characteristics that make them more likely to cause human infection than others that are more, or equally, prevalent in the environment [6]. Requirements to answer this question are; a means to subdivide the L. pneumophila population into clusters which are genetically similar so that we can describe the shared phenotypes of these clusters, and knowledge of the frequency of horizontal gene transfer (HGT) and recombination. This latter is crucial since these molecular events may result in the rapid development of novel phenotypes previously unseen in a clonal lineage and high levels of recombination may make clustering of organisms into related groups problematic [8].

Early studies using electrophoretic analysis of protein polymorphism (multi locus enzyme electrophoresis, MLEE) described 62 electrophoretic types and concluded that L. pneumophila was clonal in nature [9]. More recently a study examining four genes in the dot/icm complex [10] demonstrated clear evidence of intraspecific genetic exchange in L. pneumophila. Whilst initial studies using SBT data $[11,12]$ supported evidence for the clonal nature of L.pneumophila, it was acknowledged that intergenic recombination events could not be ruled out. Subsequent work analysing intragenic recombination in the six SBT loci and additional non-coding loci concluded that recombination was frequent in Legionella spp. [13,14]. Research which concentrated on the genes involved in the type II secretion system concluded that intragenic recombination was a rare event but there was good evidence for intergenic recombination [15]. Recent analysis that looked for recombination throughout the whole genome revealed significant levels of HGT both within the species L. pneumophila and from other GammaProteobacteria especially those, that like legionellae, are associated with amoebae [16]. A comprehensive review of the current knowledge about the population genetics, phylogenetics and genome of L. pneumophila concluded that recombination is playing a role in diversifying the species but this may have been more significant in the past than is seen with the current population of the species [17].

The EWGLI SBT database has now grown significantly since the work described in earlier publications with the addition of a seventh allele (nеuA) and the designation of Sequence Types (STs) [18]. The database contained 838 distinct sequence types at the time of this study and these were derived from strains isolated from worldwide locations in contrast to other studies that used more localised samples sets.

Therefore, in light of this large increase in novel STs, the aims of this study were;

1) To evaluate this global dataset and assess the relative contribution of recombination mediated by HGT and mutation to genome evolution.

2) To derive a method to cluster strains of similar genotype based on the type of population structure found in the first part of this study. This would provide a set of pragmatic groups that could be labelled and referred to using a common terminology within the Legionella scientific community.

3) To sequence the genomes of isolates representative of these major clusters within the population and provide an overview of the population structure. This would enable comparison of the genetic types determined by SBT with that derived by examining the diversity within the whole genome.

4) The ultimate aim was to provide a set of sequenced strains, which adequately represent the L. pneumophila pan genome. This will enable further studies where strains within a cluster are investigated in more detail, and allow testing of the hypothesis that clusters of strains are likely to share a common lineage and therefore some phenotypic similarities. 


\section{Results and Discussion}

Sequence Based Typing analysis: Recombination Tests

Choice of the best algorithm with which to cluster the sequence types of $L$. pneumophila will be informed by the population structure of the species, which will in turn be influenced by the relative contributions of recombination and mutation to sequence evolution. Therefore the frequencies of intergenic and intragenic recombination in L. pneumophila were investigated and compared to those for Staphlococcus aureus (representing a comparatively clonal species), Streptococcus pneumoniae (representing an intermediate species) and Neisseria meningitidis (representating a panmictic species).

Intergenic recombination is an event by which one allele of a locus can be replaced by another by horizontal gene transfer. The standardised index of association $\left(I_{S}^{A}\right)$ is a commonly used measure of intergenic recombination. Another measure of recombination over more than just one locus is the $\mathrm{r} / \mathrm{m}$ ratio. This is the ratio of probabilities that a base change occurs by recombination or mutation.

The results for these two tests (Table 1) are in agreement for each of the four species apart from $N$. meningitidis where the value of $I_{S}^{A}$ is anomalous being higher than that for $S$. pneumoniae. There has been the suggestion that sample bias may cause dramatic effects on the value for $I_{S}^{A}$ giving a distorted value. This effect may be diminished by including just a single example of each sequence type but the removal of many isolates can reduce the ability to estimate the extent of recombination from linkage disequilibrium [19]. Our analysis included just one example of each ST, but the $I_{S}^{A}$ value for $N$. meningitidis is still higher than would be expected. As noted by others [20,21] a high $I_{S}^{A}$ value does not necessarily infer clonality since linkage disequilibrium can still be observed in species that are highly recombining due to population structuring as observed in Helicobacter pylori for example [22]. Therefore the high value of $I_{S}^{A}$ for $N$. meningitidis may indicate a highly structured population such that the epidemic epidemiology leads to a superficially clonal population [20]. Based on these results overall L. pneumophila has intermediate levels of recombination between those of $S$. aureus and $N$. meningitidis. The value of $I_{S}^{A}$ indicates a population that tends towards being clonal, although again this may be due to a very structured population.

Based on the sequences from SBT a reticulate network tree was drawn using the Neighbor-net algorithm of SplitsTree. Reticulate networks attempt to provide a more 'explicit' representation of evolutionary history than traditional phylogenetic trees such as phylograms. They are often depicted as a phylogenetic tree with additional edges. The internal nodes in this network represent ancestral species, and nodes with more than two parents correspond to 'reticulate' events such as recombination: the
Table 1 Values of the standardised index of association and recombination to mutation ratio

\begin{tabular}{lcc}
\hline & $\begin{array}{c}\text { Standardised Index } \\
\text { of Association }\left(\mathbf{I}_{\mathrm{S}}^{\mathbf{A}}\right)\end{array}$ & $\begin{array}{c}\text { Recombination to } \\
\text { mutation ratio ( } \mathbf{r} / \mathbf{m})\end{array}$ \\
\hline $\begin{array}{l}\text { Staphylococcus aureus } \\
\text { (Clonal) }\end{array}$ & 0.193 & 1.6 \\
$\begin{array}{l}\text { Streptococcus pneumoniae } \\
\text { (Intermediate) }\end{array}$ & 0.044 & 9.3 \\
$\begin{array}{l}\text { Neisseria menigitidis } \\
\text { (Panmictic) }\end{array}$ & 0.116 & 32.5 \\
Legionella pneumophila & 0.153 & 16.8 \\
\hline
\end{tabular}

more splits in the branches seen in the resulting tree the more recombination or HGT is likely to have taken place. The SplitsTree computed from the L. pneumophila data (Figure 1) gives strong evidence for significant recombination between a subset of the lineages present within the tree and yields a highly significant phi test $(\mathrm{p}=0.0)$.

The results of the tests for examining intragenic recombination (recombination within the sequence of a gene) are summarised in Table 2. For each test the number of loci that were positive for recombination is recorded. For RDP at least two of the individual tests in the suite had to be positive in order for the locus to be scored positive overall.

Both the Sawyer's run test and RDP show L. pneumophila has an intermediate rate of intragenic recombination when compared with other bacterial species.

Overall the collected evidence from this and several previous studies $[12-14,16,17,23]$ strongly suggest that L. pneumophila is not a purely clonal organism but also undergoes significant recombination. The results presented here suggest that $L$. pneumophila retains evidence for a clonal vertical inheritance of genetic material whilst also demonstrating strong evidence of recombination by horizontal transfer of genetic loci. Although there was some evidence for recombination within the SBT genes, the frequency was low and this indicates that new alleles are most likely to be generated by point mutations rather than recombination. The signal from vertical inheritance of genetic material through clonal lineages is still evident when examining the genetic information contained from seven L. pneumophila loci. However it is also clear that recombination happens often enough so that it is a significant force in shaping the population structure. This does not alter the utility of SBT as a means to discriminate between isolates of L. pneumophila, particularly for outbreak investigation, since the results indicate that it is far from being a panmictic organism. Although we cannot infer a rate of recombination from this study, the relatively low frequency of recombination suggests that recombination would be unlikely to take place in the timescale of an outbreak and therefore the ST of isolates involved in an outbreak is also unlikely to change. 


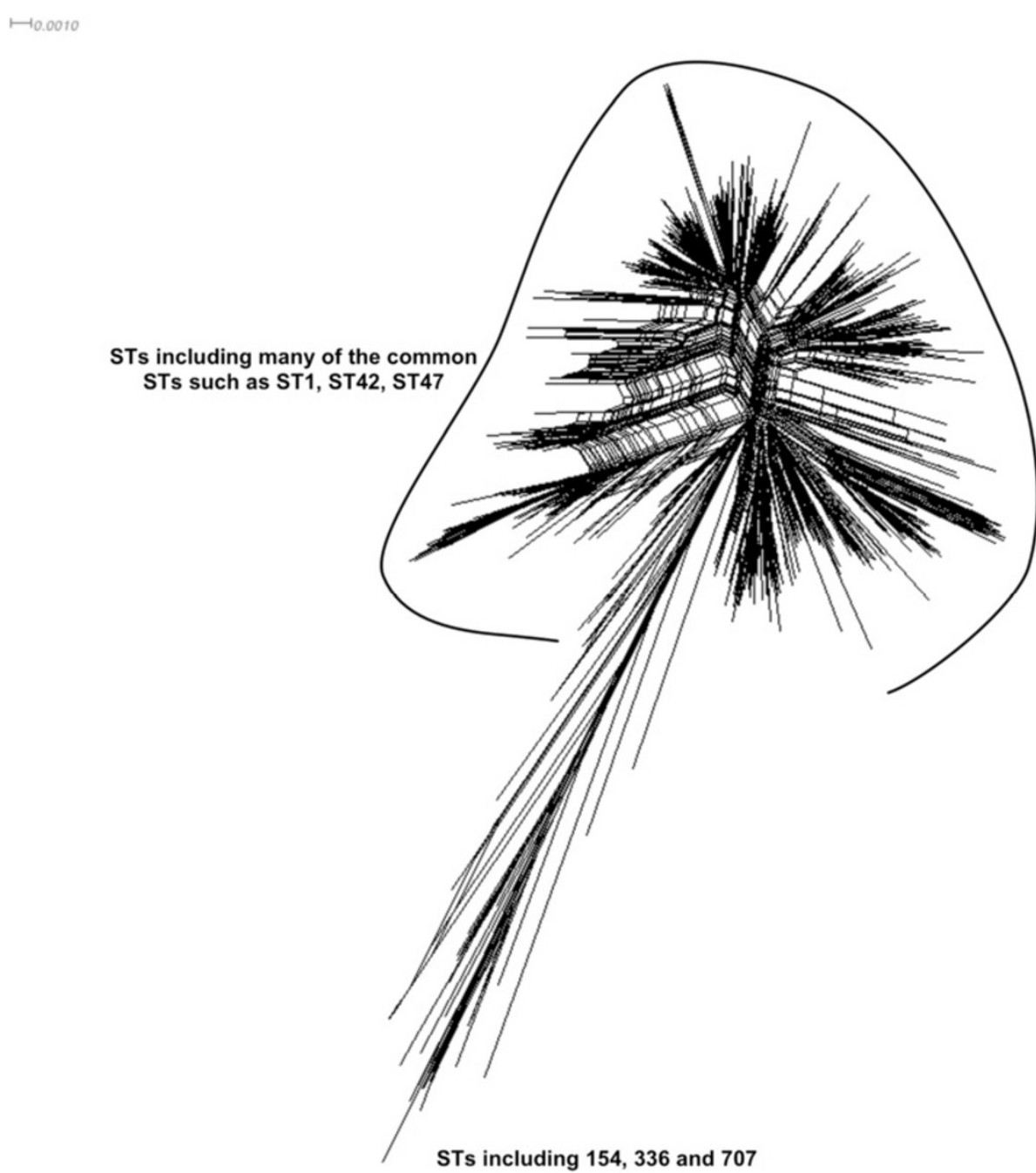

Figure 1 A reticulate tree generated by the Neighbor-net algorithm of SplitsTree4 using the concatenated sequences of the seven SBT loci as input data.

\section{Sequence Based Typing analysis: Clustering}

Since the ultimate aim of this work was to find a practical way to cluster L. pneumophila isolates, a method of determining which clustering method resulted in the most accurate sub-groups was required. Given that the recombination analysis above indicates that clonal

Table 2 Number of loci positive for recombination by the Sawyer's run test and RDP suite

\begin{tabular}{lcc}
\hline & $\begin{array}{c}\text { Sawyer's } \\
\text { run test }\end{array}$ & RDP tests \\
\hline Staphylococcus aureus (Clonal) & 0 loci & 1 locus \\
Streptococcus pneumoniae (Intermediate) & 3 loci & 4 loci \\
Neisseria menigitidis (Panmictic) & 7 loci & 6 loci \\
Legionella pneumophila & 1 locus & 2 loci \\
\hline
\end{tabular}

vertical inheritance plays a major role in the evolution of L. pneumophila, a phylogenetic tree based on the genetic distance between the concatenated sequences from the SBT loci will provide an approximate representation of the evolutionary history. Therefore a maximum likelihood (ML) tree was produced (data not shown) using RAxML [24], however bootstrapping proved computationally difficult given the sequence length and number of sequences. Therefore in order to obtain local support values for the branch split points the same data were used to produce an approximate ML tree with local support values using FastTree 2 [25]. This tree had almost identical topology to the RAxML tree and the majority of split points had local support values of $>0.8$. The same sequence data used to generate the tree were clustered using three methodologies; eBurst, BAPS of allelic data and BAPS of sequence data (Figures 2, 3 and 4). 


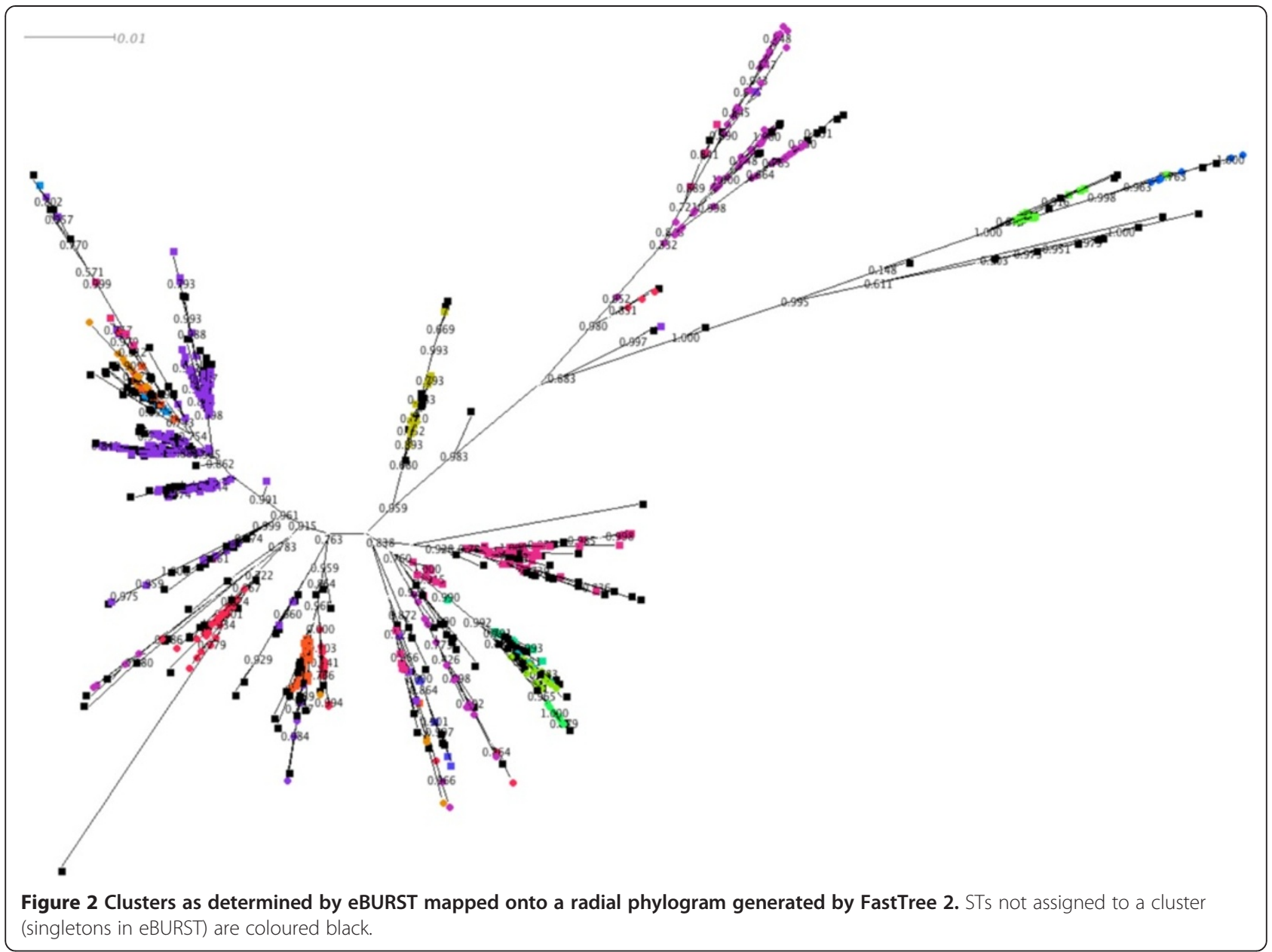

\section{eBurst analysis}

eBurst uses the BURST algorithm to identify mutually exclusive groups of related genotypes in the population, to identify the founding genotype of each group and to predict the descent from the predicted founding genotype to the other genotypes in the group [26]. The algorithm assumes that each allele is equally related to all other alleles of the same locus and as such assumes that recombination is a frequent event. eBurst clustering produced 55 groups, 31 of which contained just two STs, and 190 singletons.

\section{Bayesian Analysis of Population Structure (BAPS)}

BAPS is a tool for the detection and representation of recombination between populations [27]. The BAPS mixture model is derived using novel Bayesian predictive classification theory, applied to the population genetics context. A variety of different prior assumptions about the data can be utilized in BAPS to make inferences, however it does not require either a prior model of clonality versus recombination, or a pre-defined number of clusters. BAPS can be used to determine the population structure, to determine gene flow within a population, to determine the amount of admixture in an individual, and to divide the population into clusters $[28,29]$. The data required for BAPS population analysis can be in several formats.

The first analysis performed used allelelic data identical to that for the BURST analysis but saved in GENEPOP format. Those STs that had significant $(\mathrm{p}<0.05)$ admixture (genetic material from more than one genetic lineage) were not assigned to a cluster. With the maximum permissible number of clusters set at 20 clusters, the optimal partitioning of the $838 \mathrm{STs}$ resolved them into 15 clusters with a mean number of STs of 55.9 and a standard deviation of 48.0. However 12 sequence types had significant admixture and were excluded from clusters.

BAPS analysis was also performed using molecular sequence data. Both cluster and admixture analyses were carried out. As previously, those STs that had significant ( $\mathrm{p}<0.05$ ) admixture were not assigned to a cluster. With the maximum clusters set at 20, the optimal partitioning 


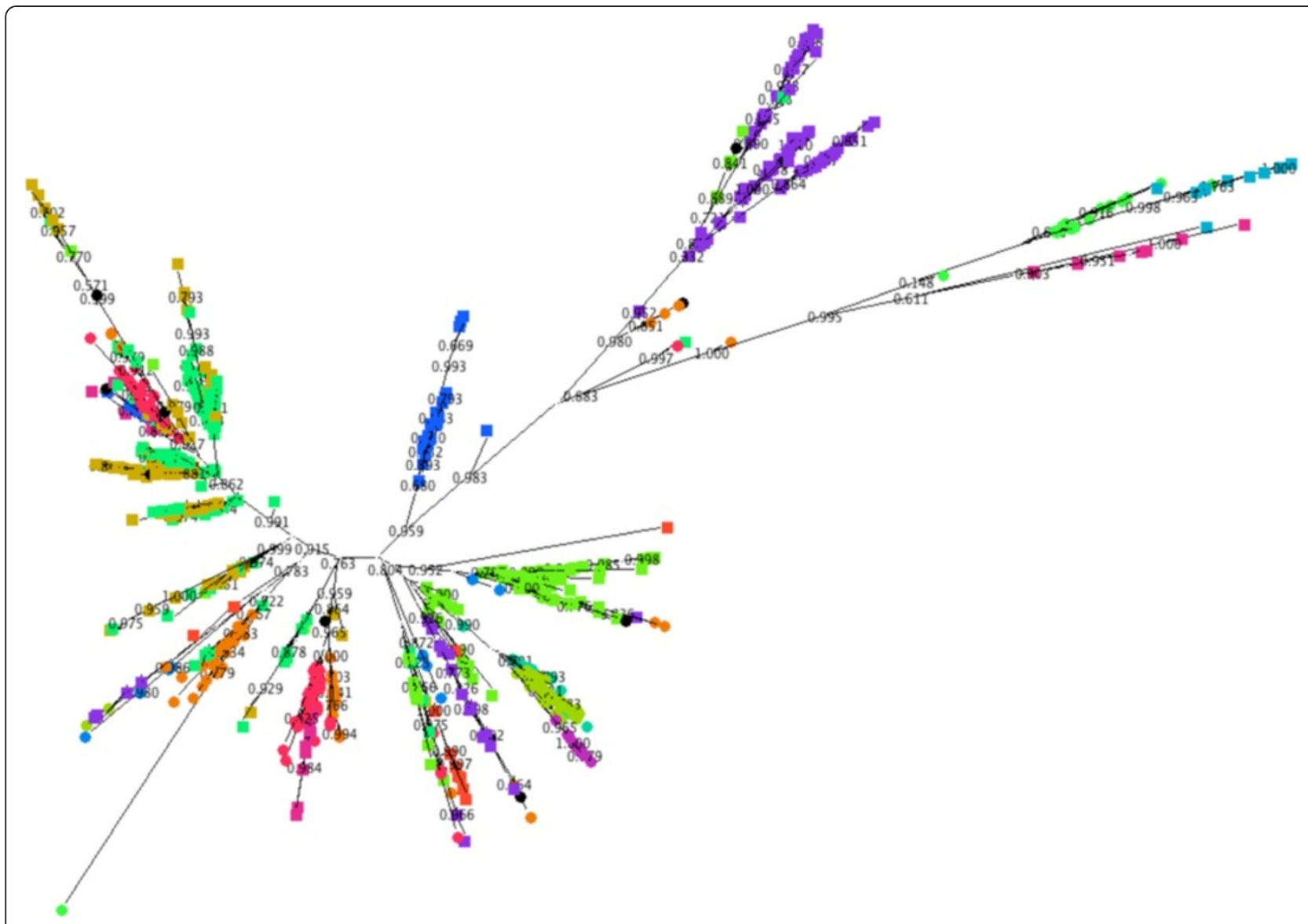

Figure 3 Clusters as determined by BAPS using allelic data mapped onto a radial phylogram generated by FastTree 2 .

of the sequence types was again found to be 15 clusters with a mean number of STs of 55.9 with a standard deviation of 31.0. However in this analysis, 181 sequence types had significant admixture and were thus excluded from clusters.

The assignment of sequence types to clusters as determined by the three methods was visualised by colouring the nodes (representing the individual STs) of a radial phylogram drawn by Dendroscope [30] according to the cluster the ST belongs to (Figures 2, 3 and 4).

By comparing different clustering methodologies we aimed to identify one that would best fit the type of population seen in the species. The data presented show that both vertical inheritance of mutation and HGT/recombination play significant roles in shaping the genetics of L. pneumophila thus an appropriate method to sub-divide the population must take both into account. It was therefore anticipated that clustering methods deriving distance between strains based on sequence identity and allowing for admixture would most accurately divide the population into clusters that reflect the true origin of the members of the cluster.
Based on the ML tree, clustering using BAPS linked sequence analysis demonstrates the most consistent mapping of clusters to the topology of the clades within the tree. On one hand this is not surprising since the BAPS analysis and ML tree both have the same input data (seven locus sequence data). However it does illustrate that clustering based on allelic data alone, and assuming linkage equilibrium, produces very different results from that when the sequence is taken into consideration: BAPS analysis using sequence data takes into account both the evolution of sequence and the flow of genetic information between populations. Therefore we consider BAPS to represent a reasonable compromise between clustering based on standard phylogenetic techniques that assume linear evolution of sequences by mutation and clustering using the BURST algorithm that assumes a freely recombining population. Based on the BAPS linked-sequence clustering 15 clusters formed the most likely partition.

\section{Genome Sequencing}

To assess if this BAPS analysis and clustering of the ST data remained valid when whole-genome data were 


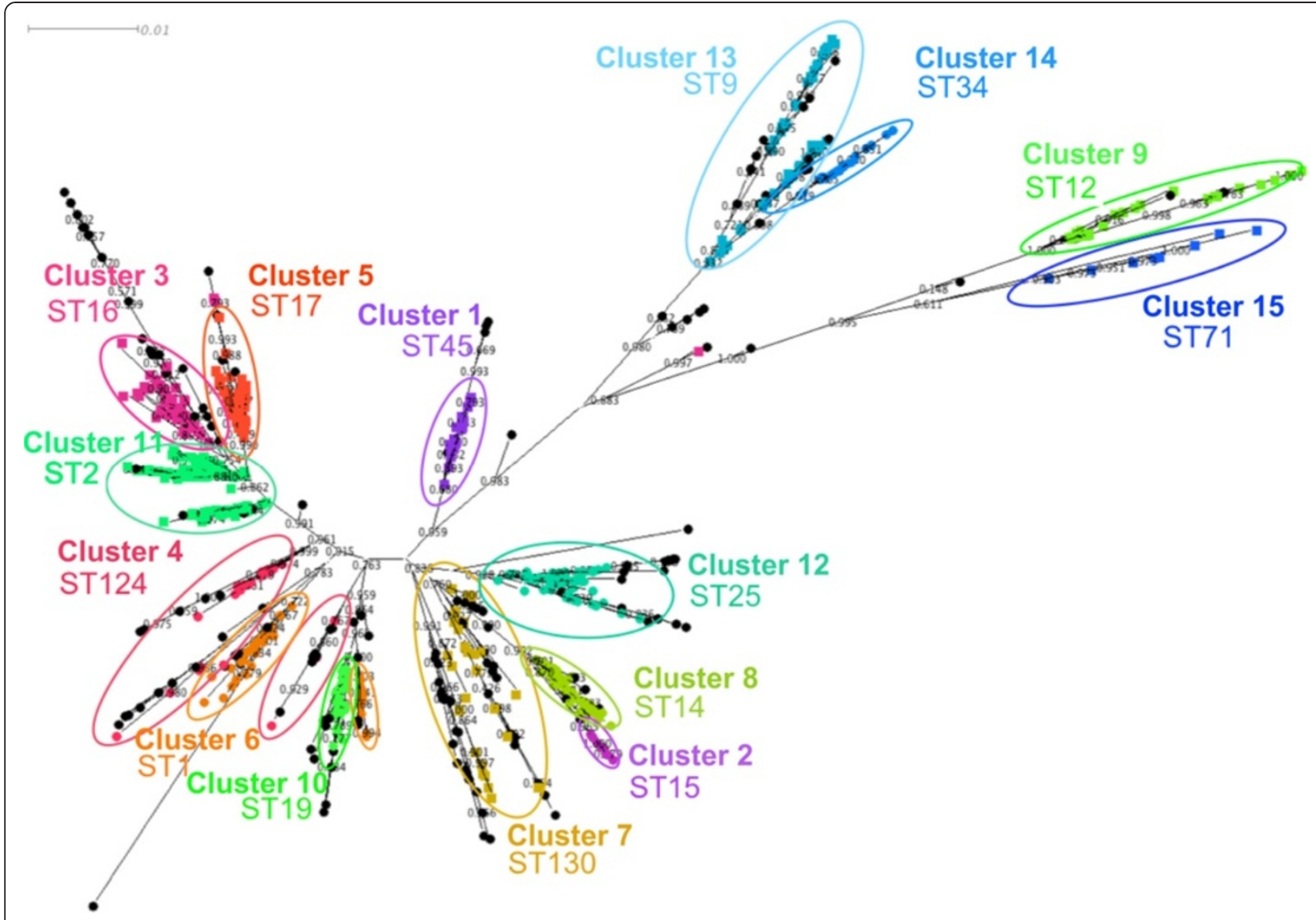

Figure 4 Clusters as determined by BAPS using linked sequence mapped onto a radial phylogram generated by FastTree 2. STs that have significant admixture are coloured black. The clusters are labelled using the lowest ST number found within the cluster.

considered, a rational approach was used to select isolates representative of each of the 15 clusters. These were sequenced using high throughput sequencing technologies (Table 3). These genomes should give a good overview of the diversity in the pan-genome of the species. The mean depth of reads using the Illumina technology is reported in Table 3. In all cases the depth was above the figure of 25 that is generally recommended for both SNP calling and de novo assembly using Illumina data.

\section{De novo assembly}

The reads were assembled de novo into scaffolds. The genomic content of these scaffolds was assessed using BLAST Ring Image Generator [37] where the scaffolds were the query sequences and the reference sequence was the genome from the Corby strain (Figure 5). Corby was chosen since it is known to be virulent in both humans and animal models and has extra mobile genetic elements not seen to date in the other sequenced legionella genomes [34,38]. Regions showing a high level of variability compared to the Corby genome were investigated further by looking at the gene content of those regions (Additional file 1: Table S1).
The graphical output from the BRIG analysis comparing the genomes to the Corby sequence displays an overview of the major regions of variability among these genomes such that 14 regions of substantial variation were observed (Figure 5 and Additional file 1: Table S1). Many of the genes present in these regions are phage or transposableelement associated, suggesting that much of this variability is driven by mobile elements. Many of these regions are adjacent to or have a tRNA sequence within them, a common location for mobile element integration [39]. Several of the variable regions have genes involved in a conjugation/type IV secretion system (T4SS). The excision, transfer and re-integration of genetic loci by this class of genes has been implicated in HGT [34]. Variability in T4SS genes has been shown previously to be a major contributor to the genome plasticity of $L$. pneumophila [23]. Other classes of genes include those encoding transporter/eflux proteins, proteins involved in glycosylation, putative virulence proteins, restriction endonuclease system proteins, and antibiotic resistance proteins. None of these proteins are involved in core metabolic functions and variability in the presence and absence of these genes is likely to result in phenotypic changes 
Table 3 Details of Legionella pneumophila strains used in the study

\begin{tabular}{|c|c|c|c|c|c|c|c|c|}
\hline ST sequenced & $\begin{array}{l}\text { BAPS } \\
\text { cluster }\end{array}$ & $\begin{array}{l}\text { Represen-tative } \\
\text { ST }\end{array}$ & Strain & Reason for selection & Strain significance & Sequence source/technology & $\begin{array}{l}\text { Mean Illumina } \\
\text { read coverage }\end{array}$ & $\begin{array}{l}\text { ENA accession } \\
\text { number or } \\
\text { reference }\end{array}$ \\
\hline 46 & 1 & 45 & H093620212 & closest to centroid & $\begin{array}{l}\text { fairly common clinical strain - } \\
\text { assumed to be virulent }\end{array}$ & NGS mate paired Illumina & 169 & ERR315646 \\
\hline $74^{*}$ & 1 & 45 & H053260229 & second of cluster & $\begin{array}{l}\text { fairly common clinical strain - } \\
\text { assumed to be virulent }\end{array}$ & NGS 454 & & ERR315647 \\
\hline $15^{*}$ & 2 & 15 & Lens & already published & caused a big outbreak in France & GenBank (NC_006369.1) & & [31] \\
\hline 84 & 2 & 15 & H043940028 & $\begin{array}{l}\text { closest available to } \\
\text { centroid }\end{array}$ & only one available from this cluster & NGS paired end Illumina & 283 & ERR315648 \\
\hline 47 & 3 & 16 & H063920004 & $\begin{array}{l}\text { internationally } \\
\text { significant }\end{array}$ & in top six strains that cause disease & $\begin{array}{l}\text { NGS 454, paired end Illumina } \\
\text { and mate paired Illumina }\end{array}$ & $\begin{array}{l}\text { paired end } 211 \text { mate } \\
\text { paired } 227\end{array}$ & ERR315649 \\
\hline 47 & 3 & 16 & Lorraine & already published & in top six strains that cause disease & GenBank(NC_018139.1) & & [23] \\
\hline 47 & 3 & 16 & LP_617 & already published & in top six strains that cause disease & EMBLBank(ERS166047) & & [32] \\
\hline $54^{*}$ & 3 & 16 & H065000139 & closest to centroid & uncommon strain nothing known & NGS paired end Illumina & 161 & ERR315650 \\
\hline 62 & 3 & 16 & H064180002 & $\begin{array}{l}\text { internationally } \\
\text { significant }\end{array}$ & in top six strains that cause disease & NGS 454 & & ERR315651 \\
\hline 611 & 4 & 124 & H090500162 & only one in cluster & unique environmental isolate & NGS mate paired Illumina & 276 & ERR315652 \\
\hline 87 & 5 & 17 & LC6677 & second of cluster & $\begin{array}{l}\text { common serogroup } 3 \text { strain - } \\
\text { does cause disease }\end{array}$ & NGS paired end Illumina & 490 & ERR315653 \\
\hline 376 & 5 & 17 & RR08000760 & closest to centroid & unique environmental isolate & NGS mate paired Illumina & 235 & ERR315654 \\
\hline $1^{*}$ & 6 & 1 & Paris & already published & & GenBank (NC_006368.1) & & [31] \\
\hline 1 & 6 & 1 & LP_423 & already published & & EMBLBank(ERS166048) & & [32] \\
\hline 5 & 6 & 1 & $\begin{array}{l}\text { EUL00013 } \\
(83 / 41091)\end{array}$ & $\begin{array}{l}\text { on an interesting } \\
\text { branch of ST001 }\end{array}$ & $\begin{array}{l}\text { only three in database - all from } \\
\text { small outbreak in Glasgow }\end{array}$ & NGS mate paired Illumina & 304 & ERR315655 \\
\hline 152 & 6 & 1 & H074360702 & closest to centroid & uncommon - mainly environmental & NGS mate paired Illumina & 180 & ERR315656 \\
\hline 179 & 7 & 130 & H093380153 & closest to centroid & uncommon but causes disease & NGS paired end Illumina & 32 & ERR315657 \\
\hline 337 & 7 & 130 & RR08000517 & second of cluster & $\begin{array}{l}\text { uncommon strain appears to be } \\
\text { phenotypically variable }\end{array}$ & NGS mate paired Illumina & 161 & ERR315658 \\
\hline 42 & 8 & 14 & $\begin{array}{l}\text { 130b } \\
\text { (Wadsworth) }\end{array}$ & already published & $\begin{array}{l}\text { in top six strains that cause disease - } \\
\text { globally distributed. Isolated } \\
\text { in USA in } \sim 1980\end{array}$ & GenBank (FR687201.1) & & [33] \\
\hline 42 & 8 & 14 & H044540088 & $\begin{array}{l}\text { internationally } \\
\text { significant }\end{array}$ & $\begin{array}{l}\text { as above but isolated in UK in } 2004 \text { - } \\
\text { assumed to be virulent }\end{array}$ & NGS 454 & & ERR315659 \\
\hline 44 & 8 & 14 & H100260089 & closest to centroid & similar to ST42 but not so common & NGS paired end Illumina & 346 & ERR315660 \\
\hline $154^{*}$ & 9 & 12 & LC677 4 & closest to centroid & $\begin{array}{l}\text { seen in Canada and UK as a cause of } \\
\text { nosocomial LD }\end{array}$ & NGS mate paired Illumina & 84 & ERR315661 \\
\hline $336^{*}$ & 9 & 12 & $\begin{array}{l}\text { Lansing-3 } \\
\text { (sgp15TS) }\end{array}$ & second of cluster & $\begin{array}{l}\text { Representative of L. pneumophila } \\
\text { subsp fraseri }\end{array}$ & NGS paired end Illumina & 150 & ERR315662 \\
\hline
\end{tabular}


Table 3 Details of Legionella pneumophila strains used in the study (Continued)

\begin{tabular}{|c|c|c|c|c|c|c|c|c|}
\hline 23 & 10 & 19 & H063280001 & closest to centroid & in top six strains that cause disease & NGS paired end Illumina & 265 & ERR315663 \\
\hline $78^{*}$ & 10 & 19 & LC6451 & second of cluster & $\begin{array}{l}\text { unique strain - caused a major outbreak } \\
\text { in Barrow in } 2002 .\end{array}$ & NGS 454 & & ERR31566 \\
\hline 51 & 11 & 2 & Corby & $\begin{array}{l}\text { already published/ } \\
\text { closest to centroid }\end{array}$ & $\begin{array}{l}\text { uncommon but well characterised - } \\
\text { virulent in animal model and protozoa }\end{array}$ & GenBank (NC_009494.2) & & [34] \\
\hline 454 & 11 & 2 & H091960011 & second of cluster & $\begin{array}{l}\text { unique environmental strain (from } \\
\text { Roman baths in Bath) }\end{array}$ & NGS mate paired Illumina & 268 & ERR315665 \\
\hline 578 & 11 & 2 & Alcoy & already published & $\begin{array}{l}\text { responsible for a very big outbreak } \\
\text { in Spain }\end{array}$ & GenBank (NC_014125.1) & & [35] \\
\hline 59 & 12 & 25 & H070840415 & closest to centroid & $\begin{array}{l}\text { quite common environmental strain - } \\
\text { a few cases of LD. }\end{array}$ & NGS mate paired Illumina & 246 & ERR315666 \\
\hline 188 & 12 & 25 & H075160080 & second of cluster & no particular data & NGS paired end Illumina & 298 & ERR31566 \\
\hline 36 & 13 & 9 & Philadelphia & $\begin{array}{l}\text { already published/ } \\
\text { closest to centroid }\end{array}$ & $\begin{array}{l}\text { the type strain - well characterised } \\
\text { caused the Philadelphia outbreak. }\end{array}$ & GenBank (NC_002942.5) & & {$[36]$} \\
\hline 37 & 13 & 9 & H034680035 & $\begin{array}{l}\text { internationally } \\
\text { significant }\end{array}$ & in top six strains that cause disease & NGS 454 & & ERR315668 \\
\hline 186 & 13 & 9 & H044500045 & second of cluster & unique clinical isolate & NGS paired end Illumina & 375 & ERR315669 \\
\hline 34 & 14 & 34 & RR08000134 & $\begin{array}{l}\text { closest available to } \\
\text { centroid }\end{array}$ & no particular significance & NGS paired end Illumina & 301 & ERR31567C \\
\hline 68 & 14 & 34 & $\mathrm{H} 074360710$ & second of cluster & no particular significance & NGS mate paired Illumina & 179 & ERR315671 \\
\hline $707^{*}$ & 15 & 71 & H091960009 & only one in cluster & $\begin{array}{l}\text { unique environmental strain } \\
\text { (from Roman baths in Bath) }\end{array}$ & $\begin{array}{l}\text { NGS paired end Illumina and } \\
\text { mate paired Illumina }\end{array}$ & $\begin{array}{l}\text { Paired end } 136 \\
\text { Mate paired } 50\end{array}$ & ERR315672 \\
\hline
\end{tabular}

Asterisks designate strains that are likely to have plasmids based on the analysis described in the methods section of this manuscript. The sequence data described can be obtained using the European Nucleotide Archive accession number listed in the table. 


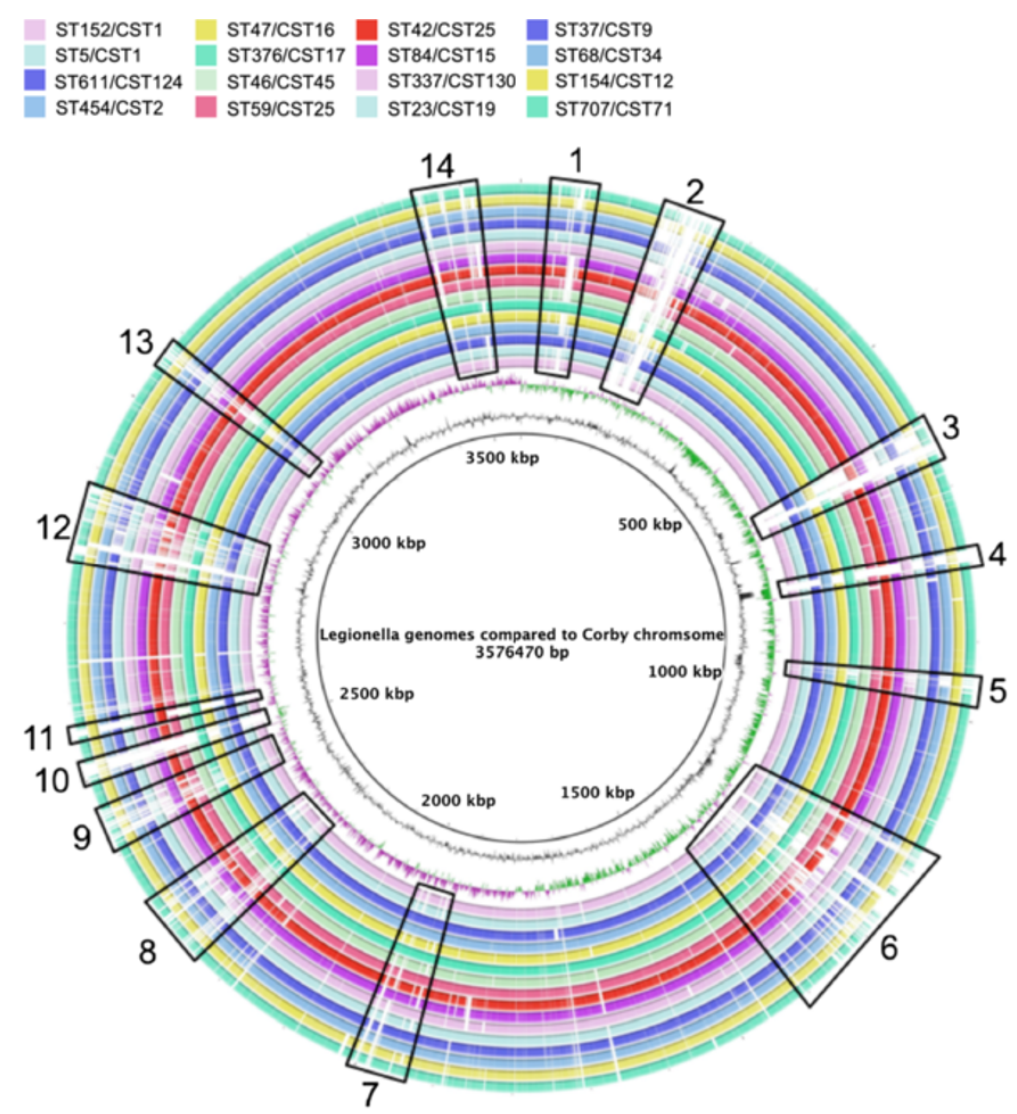

Figure 5 BRIG blast analysis of the Legionella genomes using the genome of Corby as a reference. The strains and figure colours used were from centre to outside ST152 (CST1 mauve), ST5 (CST1 light blue), ST611 (CST124 dark blue), ST454(CST2 medium blue), ST47(CST16 leaf green), ST376 (CST17 dark green), ST46(CST45 light green), ST59 (CST25 pink), ST42(CST14 red), ST84 (CST15 purple), ST337 (CST130 mauve), ST23 (CST19 light blue), ST37 (CST9 dark blue), ST68 (CST34 medium blue), ST154 (CST12 leaf green) and ST707 (CST71 dark green). Regions showing significant variability in genomic content are boxed with black lines and labelled 1-14.

that alter the ability of the organism to survive within its environment.

\section{Plasmid analysis}

Apart from acquisition of genomic islands another common way that bacteria gain genetic elements that confer phenotypic differences is by plasmid acquisition. In order to investigate the presence of plasmids in the genomes the plasmids of the Lens and Paris genomes were compared. A shared $9.2 \mathrm{~kb}$ region was used to query both the assembled and GenBank genomes. Although there may be plasmids circulating in the population that do not contain this shared locus, the same sequence is also present in the plasmid of another Legionella species, Legionella longbeachae (NSW150 plasmid pLLO: Accession FN650141) suggesting that this is a conserved sequence present in at least some of plasmids of the Legionella genus. Blast analysis detected this conserved plasmid sequence in a small proportion of the strains $(8 / 33)$ and the plasmids sequence itself was variable. The following genomes produced a hit whose e-score was less than $1 \times 10^{-20}$ : Lens: (100\% identity over 9299 bases), Paris: (83\% identity over 8319 bases), ST154: (83\% identity over 7270 bases), ST336: (83\% identity over 7270 bases), ST44: (88\% identity over 249 bases), ST54: (99\% identity over 9299 bases), ST707: (83\% identity over 7373 bases), ST74: (82\% identity over 8239 bases), ST78: (83\% identity over 7323 bases). It can be seen that there are some closely related strains (ST 154 and 336 in the same cluster) that share a very similar plasmid whereas other closely related strains (e.g. Paris, ST5 and ST152) have different plasmid content. It is likely therefore that the plasmids are exchanged horizontally between strains and have a different heredity to the chromosome. It should be noted that since the sequencing in this project is only draft sequence it is not possible to derive the complete plasmid sequences and hence their content. It is probable that the small amount of matching sequence in the ST44 strain is not from a plasmid. 


\section{Phylogeny based on gene content}

To assess variation among the genomes based on differences in gene content between the genomes, putative genes from all the genomes were grouped using cd-hit into clusters where each cluster member is homologous to one another. The clusters represent proteins shared between the genomes, and the presence of a member within these clusters for a particular strain represents the existence of the gene for this protein within the genome of that strain. There were 2173 clusters containing members from every strain sequenced (representing those genes found in all genomes) corresponding to, on average, $67.9 \%$ of the total number of genes in each genome. The mean percentage of genes shared between clusters was $85.8 \%$ (standard deviation $3.7 \%$ ) and a range of $74.8 \%$ to $98.8 \%$. The clusters were used to generate a matrix of 1 and $0 \mathrm{~s}$ corresponding to the presence or absence of a gene in each of the strains. This matrix was used as the input for a parsimony analysis, which generated a tree with the most parsimonious representation of the data (Figure 6).

\section{Phylogeny based on SNP variation}

An alternative way to assess variation among the genomes is to examine single base polymorphisms. To achieve this Illumina reads, or synthetic wgsim reads, were mapped to the Corby genome and high quality SNPs extracted for those positions conserved in all genomes. The nucleotides present in each strain at all SNP positions were concatenated and used to generate a maximum likelihood tree (Figure 7). The same SNP data was used as input for the Splits Tree program and a reticulate network tree was drawn using the Neighbor-net algorithm (Figure 8).

A number of observations are pertinent when examining these data. Two isolates (H063920004 and H091960009) were sequenced with different technologies. H063920004 with Illumina paired end, Illumina mate-paired and Roche 454, H091960009 with Illumina paired end and Illumina mate-paired. There were no SNP differences between the sequences of these replicate samples demonstrating that the protocol used for calling SNP variants is both robust and consistent. There were three isolates of ST47 (labelled ST47, LP-617 and Lorraine), two from the UK and one from France, each isolated in a different year between 2003 and 2006. These differed by just four SNPs. Two ST42 isolates, from the UK and USA (labelled ST42 and Wadsworth), were isolated 20 years apart and only exhibited 20 SNP differences. In contrast two ST1 isolates, a representative of the 'Paris' strain and a UK strain sequenced as part of another study, were isolated within 2 years of each other yet these exhibited 280 SNP differences.

These results show that lineages of L. pneumophila contain differing levels of observable diversity. There are several evolutionary scenarios that could be postulated as explanations for these observed differences. A lineage that occupies a niche where there is strong purifying selection will be less diverse. Conversely a lineage that is the result of rapid expansion within a previously unoccupied niche will tend to be more diverse. One likely scenario is that ST1 is a successful clonal lineage that emerged before the ST47 lineage and therefore has had more time to diversify by genetic drift. It is also possible that each lineage of $L$. pneumophila will be subject to differing selection pressures when infecting a human host, even though this is effectively an evolutionary dead-end. One possible scenario is that the majority of ST1 strains and a limited number of sub-lineages of ST47 cause disease in humans. If this is the case then a likely explanation is that the common ancestor of the ST1 lineage was able to infect the human species and the ancestor of the ST47 lineage did not replicate effectively in a human host. Subsequently a minority of descendents of the ST47 lineage have acquired the ability (through mutation, gene loss or acquisition) to cause human infection. Differentiating between these putative evolutionary scenarios will be difficult and will require a greater understanding of the effects of diversity within the lineages of L. pneumophila sampled from the environment and human infections.

When examining the output from the Splits Tree analysis, the more splits observed, the more recombination or HGT is likely to have taken place. The majority of clades in the tree show a branching network structure suggestive of frequent recombination. The Phi test for recombination as implemented in SplitsTree also showed evidence for recombination $(\mathrm{p}=0.0)$. The exceptions are the clade(s) containing ST136/154 and ST707. The ST136/154 clusters contain members of the sub-species L. pneumophila subsp. fraseri. This would explain the long branch length for this cluster and the genetic diversity among these strains and the rest of the population could be responsible for the low levels of horizontal exchange and recombination with the remainder of the L. pneumophila strains.

The maximum likelihood tree based on SNPs and the maximum parsimony tree based on gene presence can be used to compare clustering based on whole genome data with that based on the SBT data. In both genome trees the strains making up the majority of clusters identified by BAPS analysis of the seven SBT loci group together. This is most evident in the tree resulting from the SNP analysis. This tree and its branch lengths is mostly likely to match the true evolutionary history of the strains since, for all but the most panmictic organisms, the well understood evolutionary mechanisms causing mutations in the genome will be summarised by the SNPs occurring in positions sampled across the genome. The 


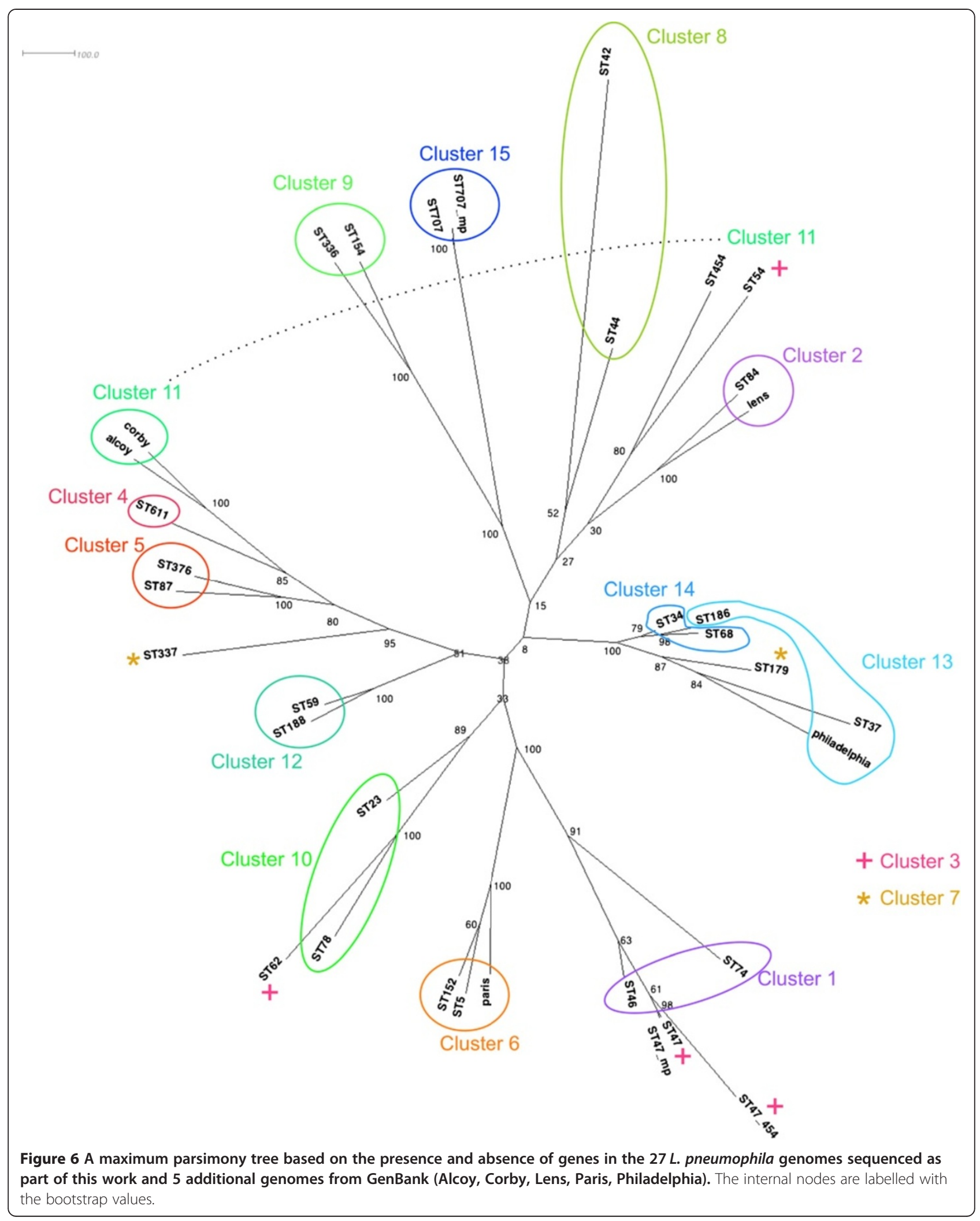




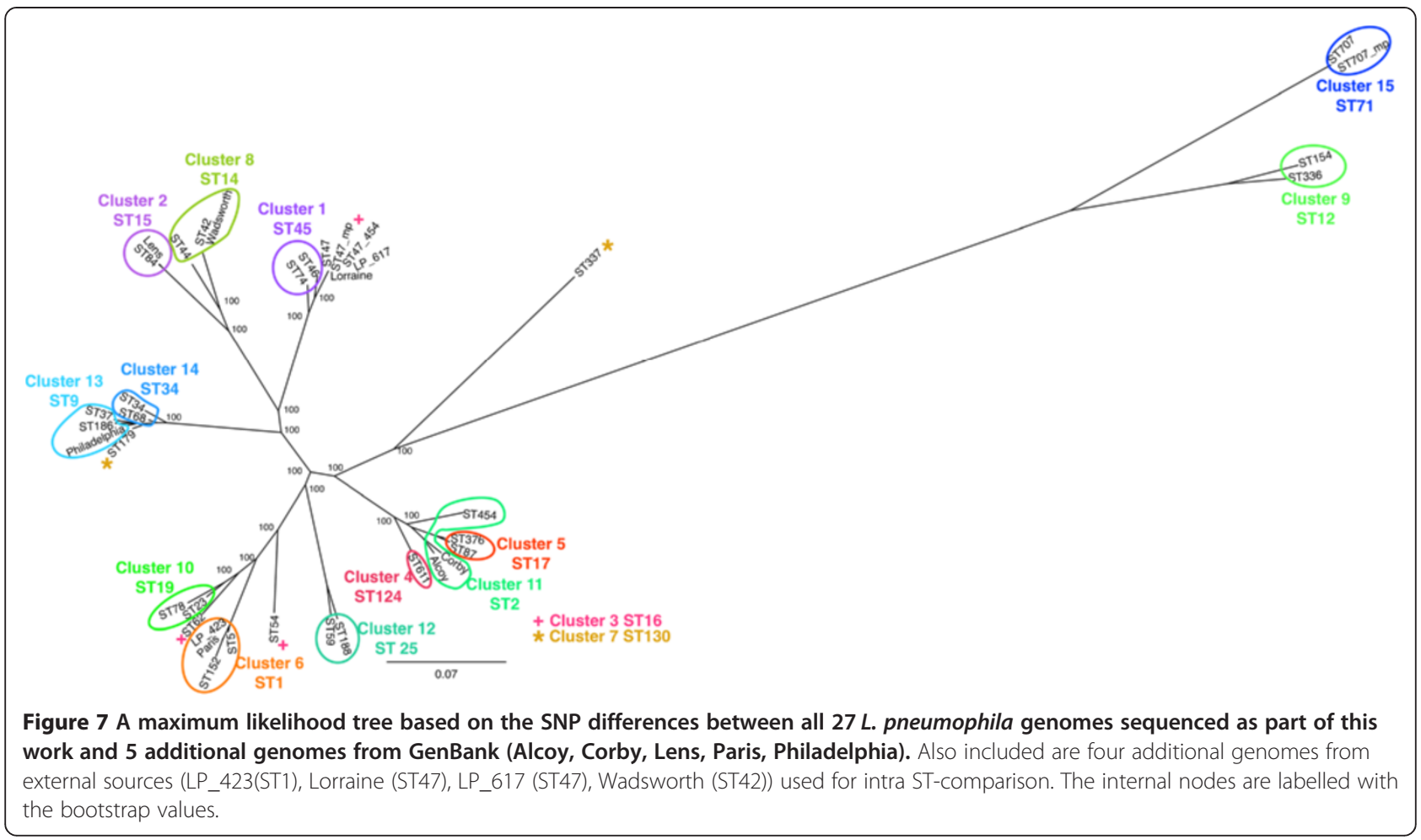

selection of core SNPs (those SNPs in locations found in all genomes) for analysis obviates the problems associated with using SNPs that are in genes that are variably present in different genomes and in loci associated with transposable elements. Some of the SNPs will be in loci that have acquired by HGT/recombination and will not match the evolutionary history of the core genome. The reason for this is that a large number of SNPs, that would have taken considerable time to arise by the process of DNA mutation, can be introduced by a single HGT event. However since $L$. pneumophila only shows moderate recombination there should be enough 'signal' from the SNPs in loci that have not undergone HGT to mask the 'noisy' data arising from SNPs arising from HGT. In the tree derived from the presence of genes in the different genomes (Figure 6) there is more evidence for strains from BAPS clusters being split over more than one branch of the tree. This is likely to be due to the fact that HGT of genes can result in large changes in presence and absence data and this tree reflects the fluid nature of the L. pneumophila genome, especially the non- core genome. One reason that may explain differences between the SBT and genomebased trees is that several of the genes that make up the SBT scheme are possibly under positive selective pressure. These include genes encoding surface proteins (flaA, mompS and pilE) and factors that may be involved in virulence (proA and mip) $[3,4]$. This is in contrast to the majority of genes in the genome which will be evolving neutrally. However although there are clear differences between the two trees, particularly in terms of the branch lengths, the overall topologies are broadly similar as measured by the groups of strains found within clades.

\section{Admixture analysis}

In both trees strains from BAPS clusters 3 and 7 are split across sometimes quite distant branches of the tree. Reexamination of the data from the original BAPs analysis shows that these results may reflect admixture of the genetic material of some of the strains from these clusters. Admixture refers to the process by which two discrete populations exchange genetic material resulting in organisms that have a genome that is sourced from two different origins.

BAPS analysis will, for each sequence, estimate the proportion of genetic material arising from organisms from each of the clusters that are derived as part of the analysis. It will also assign a p value to the likelihood of an organism being admixed. The data shows that it is likely that strains belonging to STs 47,54 , and 179 have significant admixture and that there was not enough information in the seven loci to show this when performing the initial BAPs clustering.

This hypothesis was tested further by applying the same BAPS sequence-based clustering that was originally used to generate the clusters from 838 ST to a larger dataset which became available at the end of the study 


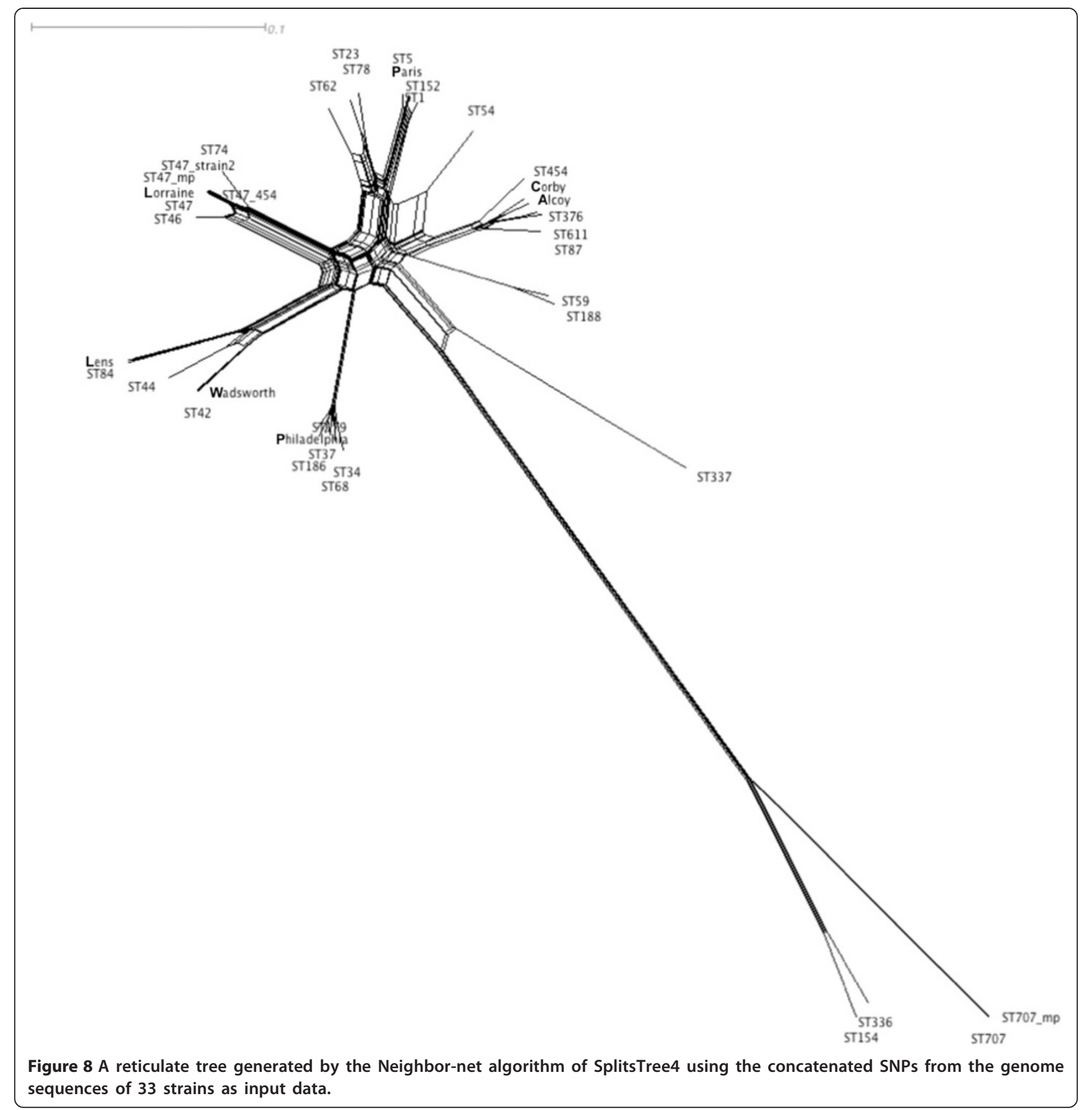

(1020 STs). These data are reported for the STs found in clusters 3 and 7 (Table 4). With the increased data available from 1020 STs the probability of these STs being admixed is now significant and it would not be possible to assign these STs to a cluster with statistical confidence. However for both ST62 and ST337 there is no significant admixture within either of the data sets and it is likely therefore that these are good representative strains for clusters 3 and 7 respectively.

In the original BAPS analysis STs 1, 5 and 152 were all assigned to cluster 6 with no significant admixture despite ST5 being in a separate clade on the phylogenetic tree derived from the seven locus sequence data. The prediction from this data was that whole genome data would show these strains to have similar ancestral origins. Both whole genome trees show this to be the case with all three STs clustering tightly in one branch of the tree.

\section{Conclusions}

This paper describes the sequencing of multiple genomes from strains representing most of the diversity present 
Table 4 Table showing admixture of Legionella pneumophila strains

\begin{tabular}{|c|c|c|c|c|c|}
\hline Cluster & ST & $\begin{array}{l}\text { Proportion of genetic material from } \\
\text { clusters ( } 838 \text { strain data set) }\end{array}$ & $\begin{array}{l}\text { Significant admixture? } \\
\text { (838 strain data set) }\end{array}$ & $\begin{array}{l}\text { Admixture analysis } \\
\text { with } 1020 \text { strains }\end{array}$ & $\begin{array}{l}\text { Significant admixture? } \\
\text { (1020 strain data set) }\end{array}$ \\
\hline 3 & 47 & 3: $0.77, \mathbf{1}: 0.21$ & no & 3: $0.36,1: 0.29,11: 0.35$ & yes \\
\hline 3 & 54 & 3: 1.0 & no & 3: $0.72,10: 0.24$ & yes \\
\hline 3 & 62 & 3: 1.0 & no & 3: 0.97 & no \\
\hline 7 & 179 & 7: $0.85,13: 0.14$ & no & 7: $0.56,13: 0.35$ & yes \\
\hline 7 & 337 & 7: 0.96 & no & 7: 1.0 & no \\
\hline
\end{tabular}

The clusters listed are those that show aberrant clustering on both trees derived from whole genome data. Only those clusters (cluster numbers shown in bold text) that contribute more than 0.1 of the genetic material of a strain are reported.

in the L. pneumophila population sampled from both environmental sources associated with human habitation and from patients with Legionnaires' disease. Based on genome comparison 2172 genes (approximately 70\% of the mean number of genes per genome) are conserved in all strains which is less than that reported previously (approximately 2400 genes, which is equivalent to $80 \%$ of the mean number of genes per genome) [16,23]. This is probably due to the samples representing a wider breadth of the population than the genomes used to calculate the core genome size in previous studies. The remaining $30 \%$ of the genome, often known as the accessory genome, is composed of many classes of genes but common themes include those that encode for functions that can mobilise DNA and those that are involved in protein transport/secretion. The former may be responsible for driving a dynamic genome in the species by permitting many mechanisms for horizontal gene transfer. The latter could be involved with niche adaptation. This and other studies have shown that recombination is a significant driver of evolution of the L. pneumophila genome. However we show that the genetic signal contained in the seven loci of the SBT scheme is generally indicative of its genomic heritage. Some STs appear to have been derived from recombination between strains of two different genetic backgrounds. However by clustering STs using BAPs we can determine which STs are likely to exhibit admixture and therefore cannot be confidently assigned to a cluster. Future studies will include looking at strains within and between clusters to determine phenotypes that are shared within a cluster but differ between clusters, and subsequently to search for the genetic differences that correlate with these phenotypes.

\section{Methods}

For L. pneumophila all STs up to and including ST850 ( $n=838$ after removing 'withdrawn' STs) were used in the study. A ST is 'withdrawn' when the depositor informs the database curators that the unique allelic profile was submitted in error and is in fact not extant. As comparator data the following MLST datasets (1 representative per ST) as present in the pubmlst.org data (July 2010 and downloaded from the links present at the URL http:// pubmlst.org/data/) were included; Staphylococcus aureus (clonal), Streptococcus pneumoniae (intermediate) and Neisseria meningitidis (panmictic).

\section{Tests for recombination}

To examine recombination within the L. pneumophila, $S$. aureus, S. pneumoniae and $N$. meningitidis populations the following types of events were tested for:

\section{Recombination between genes (intergenic)}

Three methods were used to test for this

a. Standardised Index of Association as Implemented in Start 2 [40].

b. Recombination to mutation ratio $(\mathrm{r} / \mathrm{m})$ ratio as implemented by ClonalFrame (http://www.xavierdidelot. xtreemhost.com/clonalframe.htm, [41]). The exact method used was as described by Vos et al. [42]. Parameters -x 100000 -y 100000 -z 100 -M -m (where is the Watterson estimate for the scaled mutation rate theta). This is calculated as the number of segregating sites (i.e., the number of polymorphic sites as calculated by DNAsp http://www.ub.edu/dnasp/) divided by the (n-1)th harmonic number where $\mathrm{n}$ is the number of samples.

c. SplitTree analysis. The concatenated sequences from the SBT loci for all STs were used as input for the SplitTree program (version 4.12.3) and the Neighbor-net algorithm used to draw a tree. The phi test for recombination as implemented in this program was performed.

Recombination within genes (intragenic)

Two approaches were taken

a. Running the recombination tests within the RDP3 suite[43]. A locus was considered to have undergone significant recombination if two or more of the tests in the RDP3 suite were positive.

b. Applying the Sawyer's run test (Implemented in Start 2). 


\section{Clustering algorithms eBURST}

eBURST was used to cluster strains using the default settings: grouping strains sharing alleles at $\geq 6$ of the 7 loci with at least one other ST in each group. The number of re-samplings for bootstrapping was 1000 [26].

\section{Bayesian Analysis of Population Structure (BAPS)}

This methodology is described in detail in the references [27-29]. Clustering of individuals was performed on allelic data from STs formatted in GENEPOP format. Ten runs were performed setting an upper limit of 20 clusters. Admixture analysis was performed using the following parameters: minimum population size considered 5 , iterations 50 , number of reference individuals simulated from each population 50, number of iterations for each reference individual 10. BAPS analysis was also carried out using the clustering of linked molecular data functionality. The sequence data were saved in Excel (Microsoft) format. The same parameters for clustering and admixture were used as for the allelic data.

\section{Whole genome sequencing Strains}

Strains used in the study were either sequenced by Next Generation Sequencing (NGS) technologies or available through GenBank (Table 3).

At the time of the study the EWGLI SBT database contained data from 4272 strains from 43 countries (date 09/06/2010). The authors' strain collection of strains in the database comprises 1110 clinical and environmental isolates, representing 222 ST obtained from 33 countries around the world. Although $77 \%$ of these were obtained from UK many of these STs are found worldwide and thus selecting strains only from the authors' collection is unlikely to introduce a significant geographical bias. Strains were selected from the authors' collection to represent all 15 BAPS clusters derived from SBT sequence data (Figure 4). The ST that was nearest to a notional centroid of each cluster was calculated as described below. Where possible this 'nearest to centroid' ST was used as a representative of the cluster for sequencing purposes. In all but one case, at least one other strain with a different ST from the 'centroid ST' was sequenced for each cluster. Where possible these strains were selected because the ST is of public health significance. Details are given in Table 3.

\section{Centroid of cluster calculation}

For each cluster the sequences from the seven loci that make up the SBT scheme were concatenated and a distance matrix constructed using the program dnadist from the PHYLIP suite. These distances were scaled to 2 dimensions using the multidimensional scaling function cmdscale in $\mathrm{R}$ [44] these dimensions being treated as $\mathrm{x}$ and $\mathrm{y}$ coordinates. The central coordinate in $\mathrm{x}$ and $\mathrm{y}$ space was calculated using the mean of all coordinates. The Euclidian distance of each strain in the cluster to the centroid was calculated by Pythagorean mathematics using the $\mathrm{x}$ and $\mathrm{y}$ coordinates from the multiple dimensional scaling calculations.

\section{Sequencing}

Genomic DNA from pure bacterial cultures from each of the strains was sequenced using either 454 or Illumina technologies. The strains sequenced by 454 used the titanium chemistry in conjunction with $8 \mathrm{~kb}$ insert libraries. Those sequenced employing the Illumina technology used $50 \mathrm{bp}$ read lengths in conjunction with either a paired end or mate-paired $3 \mathrm{~kb}$ insert library. Several strains were sequenced using both 454 and Illumina technologies (Table 3).

\section{Assembly}

The 454 sequences were assembled using the Newbler software (version 2.5) from Roche. Default parameters were used for assembly and scaffolding. The Illumina reads were assembled using Velvet version 1.1.05 [45]. The process was optimised using the velvet optimizer script from the Victorian Bioinformatics Consortium (https://github.com/Victorian-Bioinformatics-Consortium/ VelvetOptimiser) with a kmer range of 33 to 47 . The additional options -shortMatePaired2 yes -ins_length2 2500 -ins_length2_sd 500 were specified for reads from the $3 \mathrm{~kb}$ mate pair libraries. Contigs were joined into scaffolds using the SSPACE tool [46].

\section{Mapping and SNP calling}

In order to discover SNPs using a single method for Illumina reads, 454 reads or complete sequences from GenBank, short 'Illumina-style' reads were simulated from 454 assemblies and GenBank-derived genomes. This was achieved using the wgsim program from the Samtools package [47] with these parameters -e $0-r \quad 0-N$ $3000000-d$ 250-1 50-2 50. This resulted in two fastq files representing 3 million paired end reads of $50 \mathrm{bp}$ with an insert size of $250 \mathrm{bp}$ equivalent to the reads from the paired end libraries from the experimental Illumina sequences.

Simulated or experimental Illumina reads from all strains was mapped to the genome sequence of the Corby strain using bowtie 0.12 .7 [48] using the $-m 1$ parameter to exclude reads that map in more than one place on the reference sequence and tend to cause false positives when calling SNPs. The Sequence Alignment Map from the Bowtie mapping was sorted and indexed using samtools to produce a Binary Alignment Map (BAM). Samtools mpileup was used to create a combined Variant 
Call Format (VCF) file using each of the BAM file. The VCF file was further parsed using a simple script to extract only SNP positions that were of the high quality in all of the genomes and write out these SNPs into a multiple FASTA format file. High quality SNPs were defined as having an overall SNP quality value of $>=90$, at least one of the genomes must have a high quality (quality value $>=30)$ variant base (1/1 in the VCF file) at the SNP position, and the position must not contain SNPs reported as heterozygous (0/1 in the VCF file).

\section{Coverage}

The coverage of reads mapped to a reference genome was assessed using BEDTools (https://github.com/arq5x/ bedtools2) and the genomeCoverageBed function.

\section{Plasmid analysis}

A query sequence of 9299 bases, positions 3036 to 12334 from Lens plasmid pLPL (Accession: NC_006366) was used to search blast databases using blastall (blastn program) from NCBI.

\section{Overview of genome similarity}

BRIG (BLAST Ring Image Generator) was used to produce an image to illustrate the similarity between the Corby genome and one sequence from each of the BAPS clusters (except for Clusters 1 and 2 where two sequences were included, one from each clade on the phylogenetic tree produced from SBT data). Similarity was determined using BLASTn.

\section{Gene content analysis}

A novel method was used to cluster the genes from all the genomes in the study. This method we have termed CoreAccess is reported in full in a paper currently under preparation. Briefly, the protein sequences of all genes from the genomes were used as input for the program cd-hit [49]. These genes were either those already annotated in the sequence files of the GenBank genomes or those predicted using Glimmer3 [50] trained using the Corby sequence genes. The proteins were clustered using cd-hit using a hierarchical approach, first clustering at a high percentage cut-off and then stepwise lowering of the cut-off and clustering the clusters from the previous step. The final cut-off was $80 \%$. This hierarchical approach overcomes errors that can arise in single step clustering as described on the cd-hit website (cd-hit.org). The hypothesis underlying this methodology is that the clusters contain homologous proteins from the different genomes and as such represent groups of proteins with the same or similar function from the different genomes. In order to be able to search the clusters and find for example genes shared by all the genomes, the information about the clusters in the cd-hit output was collated into a sqlite3 database using tools within the Core Access suite.

\section{Phylogenetic Tree construction}

Maximum likelihood tree phylogenetic trees were produced from mutiple fasta files by the MEGA software package [51] using the Tamura-Nei model, and testing the phylogeny with 500 bootstrap replicates.

To construct a tree from the gene content analysis, the database generated by CoreAccess was queried using SQL so that the presence/absence of a protein representative from each strain in every cluster was recorded to produce a phylip compatible discrete state (binary $0 / 1$ ) character matrix. The seqboot program for the Phylip package [52] was used to create 100 bootstrap replicates using the Discrete Morphology data type and Non-interleaved as parameters. Applying the Phylip pars program produced 100 trees from the bootstrapped data which were subsequently fed into sumtrees [53] to result in a final consensus tree including both branch length and bootstrap values. The parameters employed were -to-newick and -no-summarymetadata. Bootstrap values were converted to a percentage value using a custom BioRuby [54] script.

\section{Additional file}

Additional file 1: Table S1. Table showing major regions of variability between the Legionella genomes as determined by blastn against the Corby genome. For each region some of the more notable features are listed.

\section{Competing interests}

The authors declare that they have no competing interests.

\section{Authors' contributions}

$\mathrm{AU}$ carried out the clustering plus whole genome sequence analysis and wrote the manuscript. GJ performed the recombination analysis and contributed to pilot clustering analyses. MM performed the laboratory work including DNA extraction and Sanger sequencing. NF coordinated the laboratory work and helped in the study design. TH conceived of the study, and participated in its overall design and coordination and helped to draft the manuscript. All authors read and approved the final manuscript.

\section{Acknowledgements}

This work was funded by the Public Health England (formerly known as Health Protection Agency).

\section{Author details}

'Bioinformatics Unit, Microbiology Services (Colindale), Public Health England, 61 Colindale Avenue, London NW9 5EQ, UK. ${ }^{2}$ Respiratory and Vaccine Preventable Bacteria Reference Unit, Microbiology Services (Colindale), Public Health England, 61 Colindale Avenue, London NW9 5EQ, UK. ${ }^{3}$ Centre for Bioinformatics, Biosciences, College of Life and Environmental Sciences, Geoffrey Pope, University of Exeter, Stocker Road, Exeter EX4 4QD, UK.

Received: 10 July 2013 Accepted: 16 December 2013

Published: 24 December 2013

\section{References}

1. Harrison TG, Saunders NA: Taxonomy and typing of legionellae. Reviews in Medical Microbiology 1994, 5:79.

2. Fry NK, Alexiou-Daniel S, Bangsborg JM, Bernander S, Castellani Pastoris M, Etienne J, Forsblom B, Gaia V, Helbig JH, Lindsay D, Christian Lück P, Pelaz C, 
Uldum SA, Harrison TG: A multicenter evaluation of genotypic methods for the epidemiologic typing of Legionella pneumophila serogroup 1: results of a pan-European study. Clin Microbiol Infect 1999, 5:462-477.

3. Gaia V, Fry NK, Harrison TG, Peduzzi R: Sequence-based typing of Legionella pneumophila serogroup 1 offers the potential for true portability in legionellosis outbreak investigation. J Clin Microbio/ 2003, 41:2932-2939.

4. Gaia V, Fry NK, Afshar B, Lück PC, Meugnier H, Etienne J, Peduzzi R, Harrison TG: Consensus sequence-based scheme for epidemiological typing of clinical and environmental isolates of Legionella pneumophila. J Clin Microbiol 2005, 43:2047-2052

5. Brehony C, Jolley KA, Maiden MCJ: Multilocus sequence typing for global surveillance of meningococcal disease. FEMS Microbiol Rev 2007, 31:15-26.

6. Harrison TG, Afshar B, Doshi N, Fry NK, Lee JV: Distribution of Legionella pneumophila serogroups, monoclonal antibody subgroups and DNA sequence types in recent clinical and environmental isolates from England and Wales (2000-2008). Eur J Clin Microbiol Infect Dis 2009, 28:781-791.

7. Vekens E, Soetens O, De Mendonça R, Echahidi F, Roisin S, Deplano A, Eeckhout L, Achtergael W, Piérard D, Denis O, Wybo I: Sequence-based typing of Legionella pneumophila serogroup 1 clinical isolates from Belgium between 2000 and 2010. Euro Surveill 2012, 17:20302.

8. Hanage WP, Fraser C, Spratt BG: Sequences, sequence clusters and bacterial species. Philos Trans R Soc Lond B Biol Sci 2006, 361:1917-1927.

9. Selander RK, McKinney RM, Whittam TS, Bibb WF, Brenner DJ, Nolte FS, Pattison PE: Genetic structure of populations of Legionella pneumophila. J Bacteriol 1985, 163:1021-1037.

10. Ko KS, Lee HK, Park M-Y, Kook Y-H: Mosaic structure of pathogenicity islands in Legionella pneumophila. J Mol Evol 2003, 57:63-72.

11. Coscollá M, Gosalbes MJ, Catalán V, González-Candelas F: Genetic variability in environmental isolates of Legionella pneumophila from Comunidad Valenciana (Spain). Environ Microbiol 2006, 8:1056-1063.

12. Edwards MT, Fry NK, Harrison TG: Clonal population structure of Legionella pneumophila inferred from allelic profiling. Microbiology (Reading, Engl) 2008, 154:852-864.

13. Coscollá M, González-Candelas F: Population structure and recombination in environmental isolates of Legionella pneumophila. Environ Microbiol 2007, 9:643-656.

14. Coscollá M, González-Candelas F: Comparison of clinical and environmental samples of Legionella pneumophila at the nucleotide sequence level. Infect Genet Evol 2009, 9:882-888.

15. Costa J, d'Avó AF, da Costa MS, Veríssimo A: Molecular evolution of key genes for type II secretion in Legionella pneumophila. Environ Microbiol 2012, 14:2017-2033.

16. Coscollá M, Comas I, González-Candelas F: Quantifying nonvertical inheritance in the evolution of Legionella pneumophila. Mol Biol Evol 2011, 28:985-1001.

17. Gomez-Valero L, Rusniok C, Buchrieser C: Legionella pneumophila: population genetics, phylogeny and genomics. Infect Genet Evol 2009, 9:727-739.

18. Ratzow S, Gaia V, Helbig JH, Fry NK, Lück PC: Addition of neuA, the gene encoding $\mathrm{N}$-acylneuraminate cytidylyl transferase, increases the discriminatory ability of the consensus sequence-based scheme for typing Legionella pneumophila serogroup 1 strains. J Clin Microbiol 2007, 45:1965-1968.

19. Hanage WP, Fraser C, Spratt BG: The impact of homologous recombination on the generation of diversity in bacteria. $J$ Theor Biol 2006, 239:210-219

20. Smith JM, Smith NH, O'Rourke M, Spratt BG: How clonal are bacteria? Proc Natl Acad Sci USA 1993, 90:4384-4388.

21. Pearson $T$, Giffard $P$, Beckstrom-Sternberg $S$, Auerbach $R$, Hornstra $H$, Tuanyok $A$, Price EP, Glass MB, Leadem B, Beckstrom-Sternberg JS, Allan GJ, Foster JT, Wagner DM, Okinaka RT, Sim SH, Pearson O, Wu Z, Chang J, Kaul R, Hoffmaster AR, Brettin TS, Robison RA, Mayo M, Gee JE, Tan P, Currie BJ, Keim P: Phylogeographic reconstruction of a bacterial species with high levels of lateral gene transfer. BMC Biol 2009, 7:78.

22. Linz B, Balloux F, Moodley Y, Manica A, Liu H, Roumagnac P, Falush D, Stamer C, Prugnolle F, van der Merwe SW, Yamaoka Y, Graham DY, Perez-Trallero E, Wadstrom T, Suerbaum S, Achtman M: An African origin for the intimate association between humans and Helicobacter pylori. Nature 2007, 445:915-918.
23. Gomez-Valero L, Rusniok C, Jarraud S, Vacherie B, Rouy Z, Barbe V, Médigue C, Etienne J, Buchrieser C: Extensive recombination events and horizontal gene transfer shaped the Legionella pneumophila genomes. BMC Genomics 2011, 12:536.

24. Stamatakis A, Ludwig T, Meier H: RAxML-III: a fast program for maximum likelihood-based inference of large phylogenetic trees. Bioinformatics 2005, 21:456-463.

25. Price MN, Dehal PS, Arkin AP: FastTree 2-approximately maximum-likelihood trees for large alignments. PLOS ONE 2010, 5:e9490.

26. Spratt BG, Hanage WP, Li B, Aanensen DM, Feil EJ: Displaying the relatedness among isolates of bacterial species - the eBURST approach. FEMS Microbiol Lett 2004, 241:129-134.

27. Corander J, Marttinen P, Sirén J, Tang J: Enhanced Bayesian modelling in BAPS software for learning genetic structures of populations. BMC Bioinformatics 2008, 9:539.

28. Corander J, Marttinen P: Bayesian identification of admixture events using multilocus molecular markers. Mol Ecol 2006, 15:2833-2843.

29. Tang J, Hanage WP, Fraser C, Corander J: Identifying currents in the gene pool for bacterial populations using an integrative approach. PLoS Comput Biol 2009, 5:e1000455.

30. Huson DH, Richter DC, Rausch C, Dezulian T, Franz M, Rupp R: Dendroscope: An interactive viewer for large phylogenetic trees. $B M C$ Bioinformatics 2007, 8:460.

31. Cazalet C, Rusniok C, Brüggemann H, Zidane N, Magnier A, Ma L, Tichit M, Jarraud S, Bouchier C, Vandenesch F, Kunst F, Etienne J, Glaser P, Buchrieser C: Evidence in the Legionella pneumophila genome for exploitation of host cell functions and high genome plasticity. Nat Genet 2004, 36:1165-1173.

32. Reuter S, Harrison TG, Köser CU, Ellington MJ, Smith GP, Parkhill J, Peacock SJ, Bentley SD, Török ME: A pilot study of rapid whole-genome sequencing for the investigation of a Legionella outbreak. BMJ Open 2013, 3:e002175.

33. Schroeder GN, Petty NK, Mousnier A, Harding CR, Vogrin AJ, Wee B, Fry NK, Harrison TG, Newton HJ, Thomson NR, Beatson SA, Dougan G, Hartland EL, Frankel G: Legionella pneumophila strain $130 \mathrm{~b}$ possesses a unique combination of type IV secretion systems and novel Dot/lcm secretion system effector proteins. J Bacterio/ 2010, 192:6001-6016.

34. Glöckner G, Albert-Weissenberger C, Weinmann E, Jacobi S, Schunder E, Steinert M, Hacker J, Heuner K: Identification and characterization of a new conjugation/type IVA secretion system (trb/tra) of Legionella pneumophila Corby localized on two mobile genomic islands. Int J Med Microbiol 2008, 298:411-428.

35. D'Auria G, Jiménez-Hernández N, Peris-Bondia F, Moya A, Latorre A: Legionella pneumophila pangenome reveals strain-specific virulence factors. BMC Genomics 2010, 11:181.

36. Chien M, Morozova I, Shi S, Sheng H, Chen J, Gomez SM, Asamani G, Hill K, Nuara J, Feder M, Rineer J, Greenberg JJ, Steshenko V, Park SH, Zhao B, Teplitskaya E, Edwards JR, Pampou S, Georghiou A, Chou I-C, lannuccilli W, Ulz ME, Kim DH, Geringer-Sameth A, Goldsberry C, Morozov P, Fischer SG, Segal G, Qu X, Rzhetsky A, et al: The genomic sequence of the accidental pathogen Legionella pneumophila. Science 2004, 305:1966-1968.

37. Alikhan N-F, Petty NK, Ben Zakour NL, Beatson SA: BLAST Ring Image Generator (BRIG): simple prokaryote genome comparisons. BMC Genomics 2011, 12:402.

38. Jepras RI, Fitzgeorge RB, Baskerville A: A comparison of virulence of two strains of Legionella pneumophila based on experimental aerosol infection of guinea-pigs. J Hyg (Lond) 1985, 95:29-38.

39. Osborn AM, Böltner D: When phage, plasmids, and transposons collide: genomic islands, and conjugative- and mobilizable-transposons as a mosaic continuum. Plasmid 2002, 48:202-212.

40. Jolley KA, Feil EJ, Chan MS, Maiden MC: Sequence type analysis and recombinational tests (START). Bioinformatics 2001, 17:1230-1231.

41. Didelot $X$, Falush D: Inference of bacterial microevolution using multilocus sequence data. Genetics 2007, 175:1251-1266.

42. Vos M, Didelot $X$ : A comparison of homologous recombination rates in bacteria and archaea. ISME J 2009, 3:199-208.

43. Martin DP, Lemey P, Lott M, Moulton V, Posada D, Lefeuvre P: RDP3: a flexible and fast computer program for analyzing recombination. Bioinformatics 2010, 26:2462-2463.

44. R Core Team: R: a Language and Environment for Statistical Computing Vienna, Austria: R Foundation for Statistical Computing; 2013.

45. Zerbino DR, Birney E: Velvet: algorithms for de novo short read assembly using de Bruijn graphs. Genome Res 2008, 18:821-829. 
46. Boetzer M, Henkel CV, Jansen HJ, Butler D, Pirovano W: Scaffolding pre-assembled contigs using SSPACE. Bioinformatics 2011, 27:578-579.

47. Li H, Handsaker B, Wysoker A, Fennell T, Ruan J, Homer N, Marth G, Abecasis G, Durbin R, 1000 Genome Project Data Processing Subgroup: The Sequence Alignment/Map format and SAMtools. Bioinformatics 2009, 25:2078-2079.

48. Langmead B, Trapnell C, Pop M, Salzberg SL: Ultrafast and memory-efficient alignment of short DNA sequences to the human genome. Genome Biol 2009, 10:R25.

49. Li W, Godzik A: Cd-hit: a fast program for clustering and comparing large sets of protein or nucleotide sequences. Bioinformatics 2006, 22:1658-1659.

50. Delcher AL, Bratke KA, Powers EC, Salzberg SL: Identifying bacterial genes and endosymbiont DNA with Glimmer. Bioinformatics 2007, 23:673-679.

51. Tamura K, Peterson D, Peterson N, Stecher G, Nei M, Kumar S: MEGA5: molecular evolutionary genetics analysis using maximum likelihood, evolutionary distance, and maximum parsimony methods. Mol Biol Evol 2011, 28:2731-2739

52. Felsenstein J: PHYLIP - Phylogeny Inference Package (Version 3.2). Cladistics 1989, 5:164-166.

53. Sukumaran J, Holder MT, Dendro P: a Python library for phylogenetic computing. Bioinformatics 2010, 26:1569-1571.

54. Goto N, Prins P, Nakao M, Bonnal R, Aerts J, Katayama T: BioRuby: bioinformatics software for the Ruby programming language. Bioinformatics 2010, 26:2617-2619.

doi:10.1186/1471-2180-13-302

Cite this article as: Underwood et al:: Comparison of the Legionella pneumophila population structure as determined by sequence-based typing and whole genome sequencing. BMC Microbiology 2013 13:302.

\section{Submit your next manuscript to BioMed Central and take full advantage of:}

- Convenient online submission

- Thorough peer review

- No space constraints or color figure charges

- Immediate publication on acceptance

- Inclusion in PubMed, CAS, Scopus and Google Scholar

- Research which is freely available for redistribution 